\title{
Nonlinear Optical Switching in Regioregular Porphyrin Covalent Organic Frameworks**
}

\author{
Bishnu P. Biswal,* Sreeramulu Valligatla, Mingchao Wang, Tanmay Banerjee, Nabil A. Saad, M. Bala \\ Murali Krishna Mariserla, Naisa Chandrasekhar, Daniel Becker, Matthew Addicoat, Irena Senkovska, \\ Reinhard Berger, D. Narayana Rao, Stefan Kaskel, Xinliang Feng*
}

\begin{abstract}
Covalent organic frameworks (COFs) have aroused immense scientific interest as an exhilarating class of porous materials due to their structure tunability and diverse properties. However, understanding of their response towards laser induced nonlinear optical (NLO) applications is in its infancy and demands prompt attention. Herein, we report three novel regioregular porphyrin based porous COFs, Por-COF-HH and its dual metalated congeners (Por-COF-ZnCu and Por-COF-ZnNi) with excellent NLO properties. Notably, intensity dependent NLO switching behavior was observed for these Por-COFs, which is highly desirable for optical switching and optical limiting devices. Moreover, the efficient $\pi$-conjugation and charge transfer transition in $\mathrm{ZnCu}$-Por-COF enable a high nonlinear absorption coefficient $(\beta=4470 \mathrm{~cm} / \mathrm{GW})$ and figure of merit (FOM = $\left.\sigma_{1} / \sigma_{o}, 3565\right)$ values compared to other state-of-art materials including molecular porphyrins $(\beta=\sim 100-400 \mathrm{~cm} / \mathrm{GW})$, metal-organic frameworks (MOFs; $\beta=\sim 0.3-0.5 \mathrm{~cm} / \mathrm{GW})$ and graphene $(\beta=900$ $\mathrm{cm} / \mathrm{GW})$.
\end{abstract}

Molecules/materials with inherent nonlinear optical (NLO) properties have profound importance in telecommunication, data

[*] Dr. B. P. Biswal, M. Wang, Dr. N. Chandrasekhar, D. Becker, Dr. R. Berger and Prof. Dr. X. Feng

Faculty of Chemistry and Food Chemistry, Center for Advancing Electronics Dresden, Technische Universität Dresden, 01062, Dresden, Germany.

Email: xinliang.feng@tu-dresden.de bishnu prasad.biswal@tu-dresden.de

Dr. S. Valligatla

Institute for Integrative Nanosciences, IFW- Dresden, 01069 Dresden, Germany.

Dr. T. Banerjee

Max Planck Institute for Solid State Research, Heisenbergstraße 1, 70569 Stuttgart, Germany.

N. A. Saad and Prof. Dr. D. N. Rao

School of Physics, University of Hyderabad, Hyderabad-500046, India.

Dr. M. B. Murali Krishna Mariserla

School of Physical Sciences, Central University of Karnataka,

Kadaganchi -585367 , India.

Dr. M. Addicoat

School of Science and Technology, Nottingham Trent University Clifton Lane, NG11 8NS Nottingham (UK).

Dr. I. Senkovska and Prof. Dr. S. Kaskel

Chair of Inorganic Chemistry, Technische Universität Dresden, 01062, Dresden, Germany.

[**] This project has received funding from the European Union's Horizon 2020 research and innovation programme under grant agreement No 696656 (Graphene Flagship) and was supported financially by the Center for Advancing Electronics Dresden (cfaed), the European Social Fund, and the Federal State of Saxony (ESFProject "GRAPHD", TU Dresden). We thank Ms. Viola Duppel for SEM and TEM images, Ms. Kerstin Gottschling for TGA, Ms. MarieLuise Schreiber for elemental analysis, Prof. Ulrich Starke and Dr. Kathrin Müller for XPS data, Dr. Xiaodong Zhuang and Dr. Renhao Dong for helpful discussions.

Supporting information for this article is available on the WWW under http://www.angewandte.org or from the corresponding author. storage, display technologies, sensors and in bio-medical devices. ${ }^{[1]}$ In this regard, molecular porphyrins have been widely studied due to their versatile optical and electrochemical properties, large and fast NLO responses, possibility of incorporating a wide range of metals and their good thermochemical stabilities for optical limiting and optical switching applications. ${ }^{[2]}$ Nonetheless, to enhance and tune optical nonlinearities of singular porphyrin moieties, bathochromic shifting of their absorption bands by large m-electron delocalization are crucial, which can be possible through de novo design of integrated porphyrin units with extended $\pi-$ conjugations. ${ }^{[3][4]}$ Along this line, we propose, porphyrin-linked covalent organic framework (COF $)^{[5]}$ as model system, wherein enhancement and switching of the NLO response can be studied by manipulation of the framework. Among the recognized crystalline materials, COFs belongs to most recent class, known for being mechanically robust and offering high accessible surface area. By virtue of their freedom to be structurally and electronically tuned, so far COFs have engrossed particular interest in research areas like adsorption/storage, ${ }^{[6]}$ chemical sensors, ${ }^{[7]}$ electronics ${ }^{[8]}$ and catalysis. ${ }^{[9]}$ Hitherto, despite having potential, none of the COFs composed of porphyrin units have been explored as NLO materials. For this purpose, a regioregular ordering of multiple metal centres in a crystalline, 2D porphyrin framework, prefereably without a post-synthetic modification is desirable, which has been a difficult task ${ }^{[10]}$ and prospect to have large demand for applications in optoelectronics and photo/electrocatalysis.

In this work, we demonstrated a conjugated imine linked porphyrin-homopolymeric COF (Por-COF-HH) by Schiff base A4B4 condensation of 5,10,15,20-tetrakis(4-formylphenyl)$21 \mathrm{H}, 23 \mathrm{H}$-porphyrin (TFPP) and 5,10,15,20-tetrakis(4aminophenyl)-21H,23H-porphyrin (TAPP) (Figure 1). Adopting the same pathway, we also synthesized regioregular multimetalloporphyrin COFs with two different metal centres such as $\mathrm{Zn}, \mathrm{Cu}$ (Por-COF-ZnCu) and $\mathrm{Zn}, \mathrm{Ni}$ (Por-COF-ZnNi) respectively imbedded within a solitary framework. These porphyrin COFs (Por-COFs) are highly crystalline and porous with a BrunauerEmmett-Teller (BET) surface area up to $\sim 1300 \mathrm{~m}^{2} \mathrm{~g}^{-1}$, which are well comparable with the porphyrin COFs reported in the literature. ${ }^{[5]}$ Further, we have evaluated the NLO properties of these Por-COFs by the open aperture Z-scan technique at 532 $\mathrm{nm}$ excitation. ${ }^{[1 \mathrm{c}, 11,13]}$ Interestingly, on one hand, these Por-COFs shows optical switching behaviour from saturable absorption (SA) to reverse saturable absorption (RSA) along increase in the input laser intensity and on the other hand metallated Por-COFs display high nonlinear absorption coefficient values $(\beta=\sim 4500$ $\mathrm{cm} / \mathrm{GW}$ ), which could play potential role in photonics and optical limiting devices.

The Por-COF-HH was synthesized using an optimized solvothermal protocol; in brief, 5,10,15,20-tetrakis(4aminophenyl)-porphyrin (TAPP) and 5,10,15,20-tetrakis(4formylphenyl)-porphyrin (TFPP) are reacted in the presence of 
a)

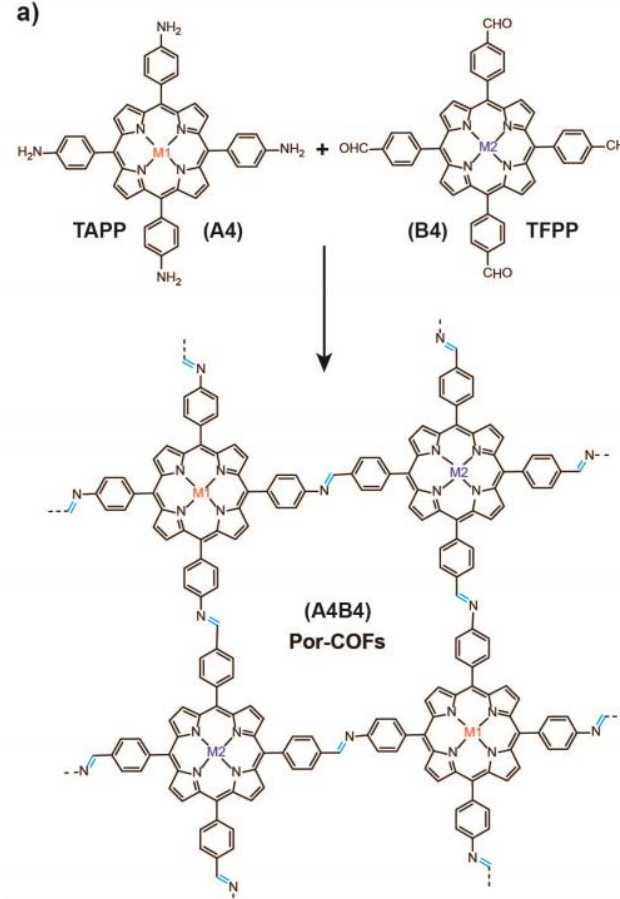

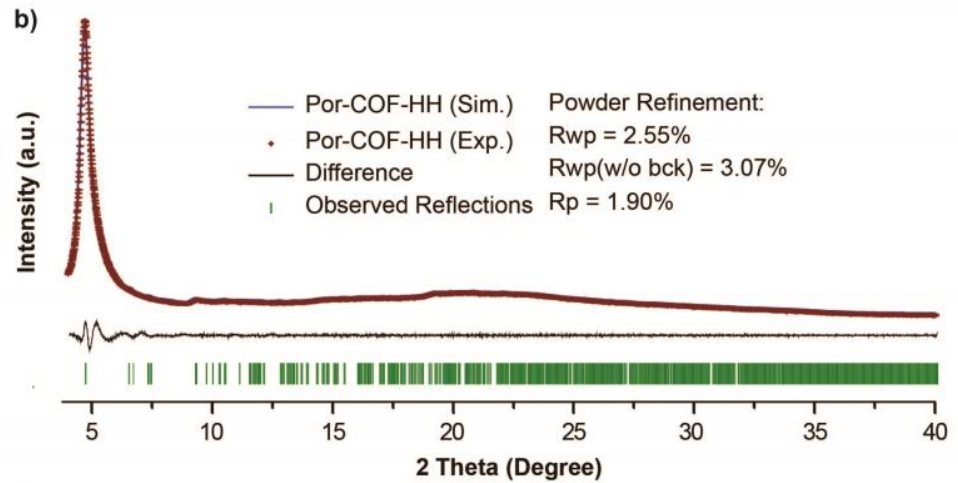

c)

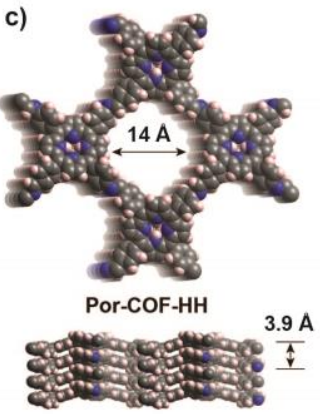

2 Theta (Degree)
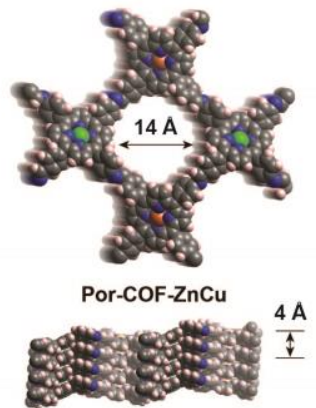

Por-COF-ZnCu

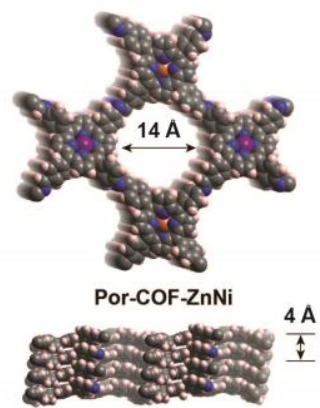

Por-COF-HH (M1 \& M2 = H); Por-COF-ZnNi (M1 = Zn; M2 = Ni); Por-COF-ZnCu (M1 = Zn; M2 = Cu)

Figure 1. a) Schematic representations of the synthesis of Por-COFs (Por-COF-HH, Por-COF-ZnCu and Por-COF-ZnNi; b) Experimental (red) compared with Powder refined (blue) PXRD profiles of Por-COF-HH with eclipsed (AA) arrangement; difference plot is given in black and observed reflections in green); c) The packing models of corresponding Por-COFs indicating the pore aperture and $\pi-\pi$ stacking distances (Zn: orange; Cu: green and Ni: purple).

aqueous acetic acid (6M) using o-dichlorobenzene and $n$-butanol (1:1 by vol.) as the solvent combination in a Pyrex tube at $120{ }^{\circ} \mathrm{C}$ for 3 days. The same protocol was employed to synthesize PorCOF-ZnCu and Por-COF-ZnNi using $\mathrm{A} 4$ and $\mathrm{B} 4$, metalloporphyrins as detailed in Section S2, Supporting Information. The synthesized Por-COFs were first examined via powder $X$-ray diffraction (PXRD) to investigate the crystallinity and phase purity. As revealed from PXRD, all the Por-COFs (Por-COF-HH, PorCOF-ZnCu and Por-COF-ZnNi) show good crystallinity with the first and most intense peak at a low $2 \theta$ angle of $\sim 4.7^{\circ}$ corresponding to the (100) reflection planes, along with other minor peaks (Figure 1b). Based on the initial experimental PXRD patterns, 2D COF models were built and optimized using the SelfConsistent Charge Density Functional Tight-Binding (SCC-DFTB) method. ${ }^{[12 a]}$ The experimental PXRD patterns match fairly well with the simulated ones derived from the eclipsed (AA) stacking models (Figure $1 \mathrm{~b}$ and Figure S6-8). The centre-to-centre distance (pore aperture) is $\sim 1.4 \mathrm{~nm}$ and the $\Pi-\pi$ stacking distances between the individual layers were calculated as $\sim 4 \AA$ for all three Por-COFs (Figure 1c). The latice parameters of PorCOF-HH were calculated to be $a=27.32 \AA, b=26.60 \AA, c=7.96$ $\AA, \alpha=88.51^{\circ}, \beta=89.83^{\circ}$ and $\gamma=89.76^{\circ}$ using the Pawley refinement on Reflex module of BIOVIA Materials Studio 2017 (Figure 1b). The refinement details for Por-COF-ZnCu and PorCOF- $\mathrm{ZnNi}$ are presented as Figures $\mathrm{S} 9$ and $\mathrm{S} 10$.

The formation of the Por-COFs by the Schiff-base condensation reaction was evidenced by the almost complete disappearance of the $-\mathrm{N}-\mathrm{H}$ stretching bands $\left(3100-3400 \mathrm{~cm}^{-1}\right)$ of TAPP, Zn-TAPP; and the $-\mathrm{C}=\mathrm{O}$ stretching bands $\left(\sim 1695 \mathrm{~cm}^{-1}\right)$ of TFPP, Cu-TFPP and Ni-TFPP; and appearance of a new characteristic $-\mathrm{C}=\mathrm{N}$ stretching band $\left(\sim 1625 \mathrm{~cm}^{-1}\right)$ in the Fouriertransform infrared (FT-IR) spectrum of Por-COFs (Figures S11$\mathrm{S} 13, \mathrm{ESI})$. The residual $-\mathrm{N}-\mathrm{H}$ and $-\mathrm{C}=\mathrm{O}$ stretching signals
(Figures S11-13) possibly correspond to the uncondensed functional groups at the edges/termini of the COF crystallites. The ${ }^{13} \mathrm{C}$ cross-polarization magic-angle spinning (CP-MAS) NMR spectroscopy of Por-COFs confirms the chemical structure of the backbone by showing the characteristic chemical shift of the $\mathrm{C}=\mathrm{N}$ carbon at $\sim 160 \mathrm{ppm}$, along with characteristic chemical shifts attributed to the porphyrin units. The full assignment is presented in Figure 2a and Figure S14. Transmission electron microscopy (TEM) images revealed significant crystallinity of the COF samples with well-defined, square shaped crystallites with average sizes between 50-100 nm (Figure 2b-d). Tetragonal pores of $\sim 1.5 \mathrm{~nm}$ were clearly visible which is close to the calculated pore size. SAED analysis shows a prominent diffraction ring at $\sim 1.8 \mathrm{~nm}$ corresponding to the (100) reflection for all three Por-COFs. A similar morphological feature was also observed in scanning electron microscopy (SEM) images, wherein cubic shape crystals of Por-COFs can be clearly visualized (Figure S16). Interestingly, for Por-COF-HH and PorCOF-ZnNi the crystallite sizes are close to $100 \mathrm{~nm}$, while for PorCOF-ZnCu, the crystallite sizes are $\sim 50 \mathrm{~nm}$. Moreover, Energy Dispersive X-Ray Analysis (EDX) confirms the presence of two different metals within the single framework of Por-COF-ZnCu and Por-COF-ZnNi (Figure S17-S19). We have also verified the individual metal content in the Por-COF- $\mathrm{ZnNi}$ and $-\mathrm{ZnCu}$ frameworks using induced coupled plasma (ICP) analysis (Section S2). X-ray photoelectron spectral (XPS) analysis validates our proposed Por-COF- $\mathrm{ZnNi}$ and $-\mathrm{ZnCu}$ structures wherein metal atoms have been successfully integrated in the COF backbone (Figure S21). Thermogravimetric analysis (TGA) was performed for all three Por-COFs under both $\mathrm{N}_{2}$ and air, to study the thermal stability and to estimate roughly the total metal content (Figure S22 and S23). 
a)

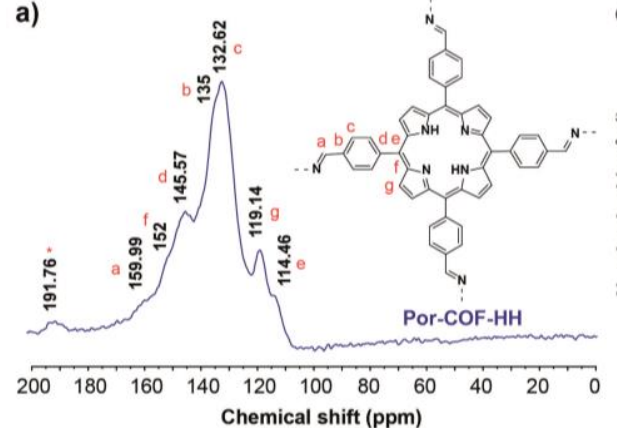

b)

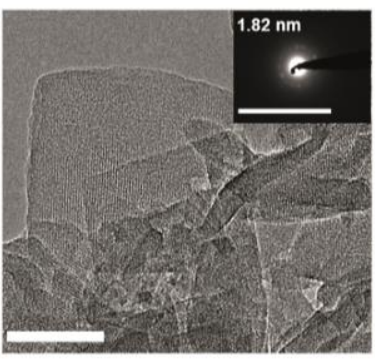

C) Por-COF-ZnNi

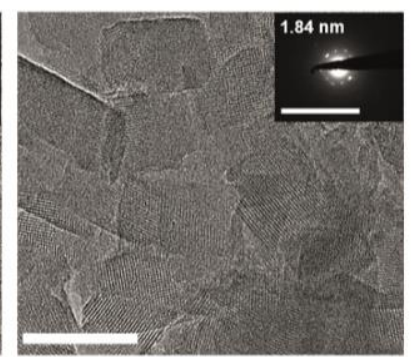

e)

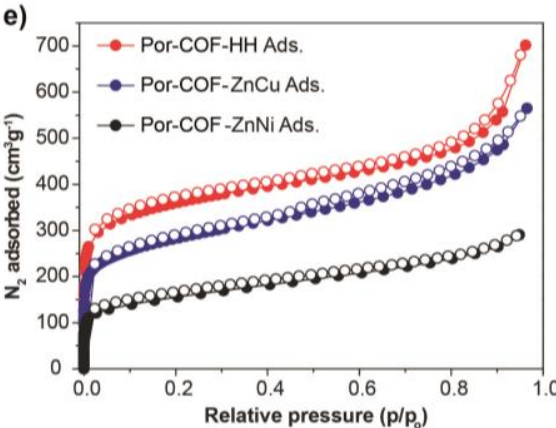

d) Por-COF-ZnCu

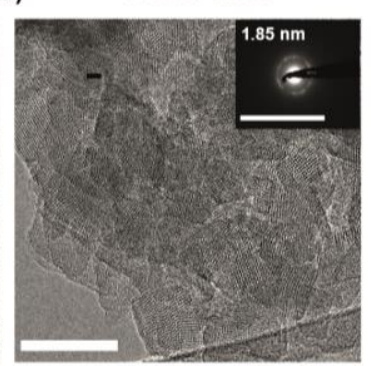

f)
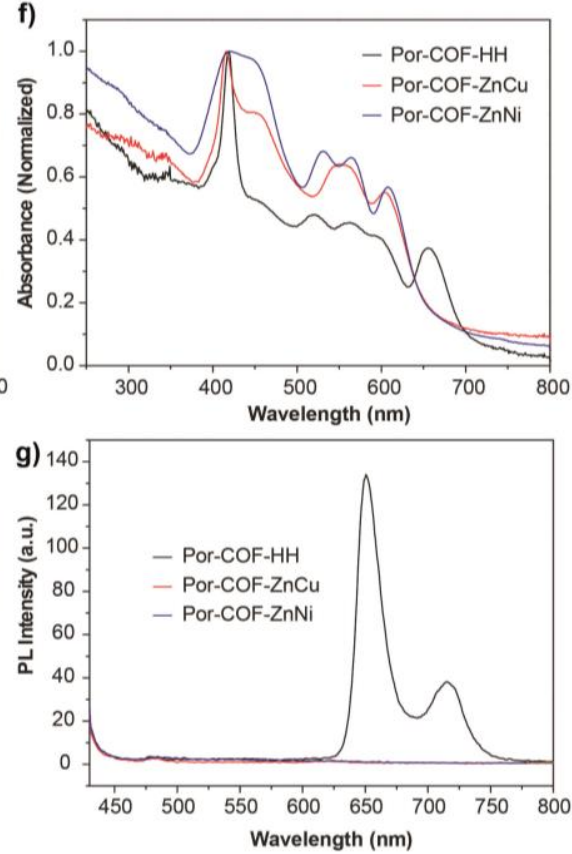

Figure 2. a) Representive ${ }^{13} \mathrm{C}$ cross-polarization magic-angle spinning (CP-MAS) solid state NMR spectra of Por-COF-HH ( ${ }^{*} \mathrm{Corresponds}$ to peripheral $-\mathrm{CHO}$ groups); b, c and d) TEM images of Por-COF-HH, Por-COF-ZnNi and Por-COF-ZnCu respectively (The main scale bars correspond to $60 \mathrm{~nm}$ in all images and the scale bar in the insets correspond to $1 / 0.37 \mathrm{~nm}$ ); e) $\mathrm{N}_{2}$ physisorption isotherms at $77 \mathrm{~K}$; Photophysical properties of Por-COFs: $\mathrm{f}$ ) UV-Vis spectra (measured in the integration sphere in dispersion state in 2-propanol) and g) Photoluminescence (PL) spectral profiles.
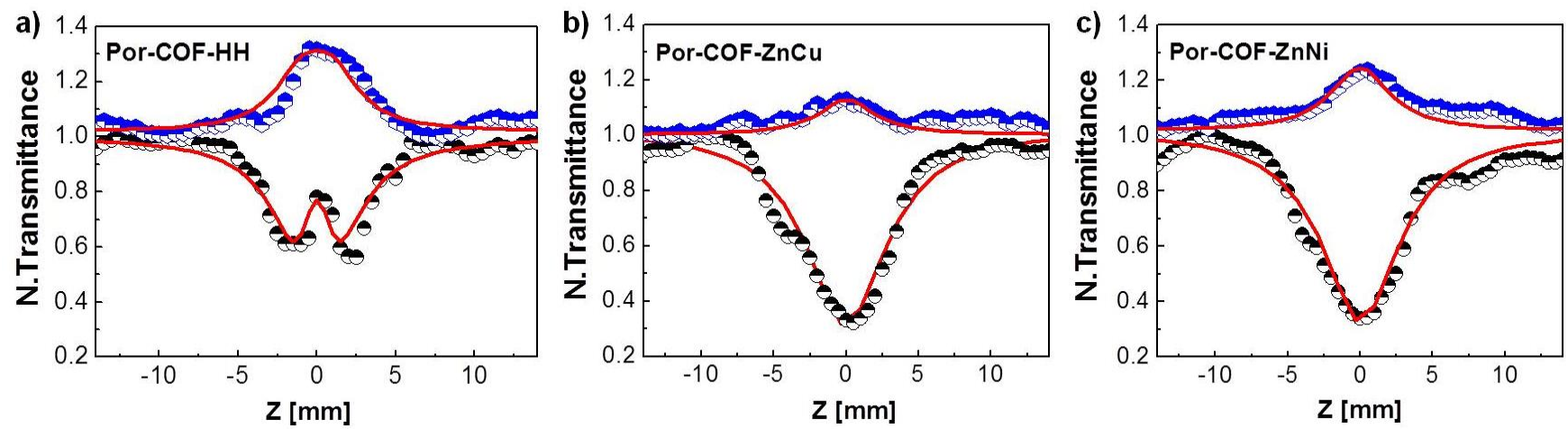

Figure 3. Open aperture Z-scan traces for Por-COFs: a) Por-COF-HH; b) Por-COF-ZnCu; and c) Por-COF-ZnNi at the excitation of 532 nm, ns laser at the input intensity of $0.25 \mathrm{GW} / \mathrm{cm}^{2}$ (represented by circles) and $0.01 \mathrm{GW} / \mathrm{cm}^{2}$ (represented by hexagons). The solid lines represent the theoretical fitting, spheres and hexagons are the experimental data

Permanent porosity of these Por-COFs was evaluated by employing $\mathrm{N}_{2}$ adsorption-desorption experiments at $77 \mathrm{~K}$. All three Por-COFs show similar isotherm shape with a steep increase in adsorption amount below 0.1 bar, characteristic for the filling of the micropores (Figures $2 \mathrm{e}$ ). The surface areas of the Por-COFs derived from the $\mathrm{N}_{2}$ adsorption data using the BET model are $1316 \mathrm{~m}^{2} \mathrm{~g}^{-1}$ for Por-COF-HH, $1027 \mathrm{~m}^{2} \mathrm{~g}^{-1}$ for PorCOF-ZnCu and $551 \mathrm{~m}^{2} \mathrm{~g}^{-1}$ for Por-COF-ZnNi, respectively. The acsessible geometrical surface areas calculated by Poreblazer software ${ }^{[12 b]}$ amount to $1512 \mathrm{~m}^{2} \mathrm{~g}^{-1}$ for Por-COF-HH, to $1457 \mathrm{~m}^{2} \mathrm{~g}$ 1 for Por-COF-ZnCu and to $1479 \mathrm{~m}^{2} \mathrm{~g}^{-1}$ for Por-COF-ZnNi. The significantly lower surface area value measured for the PorCOF- $\mathrm{ZnNi}$ is possibly due to its moderate crystallinity in comparison to others. The pore size distribution plots of Por$\mathrm{COF}-\mathrm{HH}$, Por-COF-ZnNi and Por-COF-ZnCu (Figure S24) calculated from $\mathrm{N}_{2}$ adsorption data using Quenched Solid State Density Functional Theory (QSDFT) model and cylindrical-slit adsorption kernel for carbon show the maxima centered between 1.0 and $1.3 \mathrm{~nm}$. It corresponds well to the values obtained by Poreblazer $(1.2 \mathrm{~nm}$ for pore limiting diameter, 1.3 $\mathrm{nm}$ for maximum pore size)

To understand the optoelectronic properties of the synthesized Por-COFs, we performed UV-Vis absorption in the centre mount position of an integrating sphere in the dispersion state (2-propanol) and photoluminescence measurements. The photo-physical properties of Por-COF-HH drastically differ compared to the corresponding metalated counterparts, PorCOF-ZnCu and Por-COF-ZnNi (Figure $2 f$ and $2 \mathrm{~g}$ ). The UV-Vis spectral studies display an obvious shift in all four Q-band components [500-700 nm: Qy $(1,0), \operatorname{Qy}(0,0), Q x(1,0), Q x(0,0)]$ 
of Por-COF-HH, in terms of their positions and intensity ratios compared to its metalated counterparts. Whereas, in the case of Soret B-band $(\mathrm{B}(0,0))$ of Por-COF-HH associated to two components [401 (shoulder) and $418 \mathrm{~nm}$ (dominant major peak)] and the corresponding metalated ones (Por-COF-ZnCu and PorCOF-ZnNi) exhibit only one peak (417 and $419 \mathrm{~nm}$ respectively) (Figure 2f). Moreover, the broadening of the Soret B-band was noticed in the metalated Por-COFs compared to the metal-free Por-COF. These minor changes can be attributed to the metalation of the porphyrin network and associated structural changes. ${ }^{[13]}$ In addition, we also observed photoluminescence (PL) quenching for both Por-COF- $\mathrm{ZnCu}$ and Por-COF- $\mathrm{ZnNi}$, whereas the metal-free Por-COF-HH shows reasonable emission at $650 \mathrm{~nm}$ (Figure $2 \mathrm{~g}$ ). The estimated excited-state lifetimes are $\tau_{\mathrm{avg}}=9.17 \mathrm{~ns}, 1.77 \mathrm{~ns}$ and $1.73 \mathrm{~ns}$ for Por-COF-HH, $\mathrm{COF}-\mathrm{ZnCu}$ and Por-COF- $\mathrm{ZnNi}$ respectively measured in the dispersion state (2-propanol) employing time-correlated singlephoton counting (TCSPC) method (Figure S25).

The NLO properties of Por-COFs were investigated by the open aperture Z-scan technique at the excitation wavelength of $532 \mathrm{~nm}$ from frequency doubled Nd: YAG laser with different input intensities. The Z-scan measurements with intensities ranging from 0.01 to $0.25 \mathrm{GW} / \mathrm{cm}^{2}$ reveal that nonlinear absorption mechanism is purely intensity dependant. Interestingly, all the Por-COF samples exhibited NLO switching behaviour as the nonlinear absorption swap from saturable absorption (SA) to reverse saturable absorption (RSA) upon increasing input intensity. Figure 3 represents the open aperture Z-scan curves for a) HH Por-COF-HH, b) Por-COF- $\mathrm{ZnCu}$, and c) Por-COF- $\mathrm{ZnNi}$ at the peak intensities of 0.01 and 0.25 $\mathrm{GW} / \mathrm{cm}^{2}$. Notably, at the lower intensity of $0.01 \mathrm{GW} / \mathrm{cm}^{2}$ all PorCOFs show SA behavior. Remarkably, as the input intensity increases, in Por-COF-HH, the nonlinear absorption behavior switches from RSA to SA and then again back to RSA. This RSA-SA-RSA switching behavior can be attributed to the dominant excited state absorption at lower intensities (far from focus) and saturation of excited states at higher intensity (near the focus). Whereas, in the case of Por-COF-ZnCu and PorCOF-ZnNi, excited state absorption leads to characteristic RSA behavior. To explain these results, we employed theoretical modelling based on five level rate equations as explained in the literature for metal-porphyrins (Section S5). ${ }^{[14]}$ In Figure 3, redlines are the best theoretical fits to the experimental data at different input intensities. From the fit, we deduced the nonlinear absorption coefficient $(\beta)$, ground state absorption cross section $\left(\sigma_{0}\right)$, first excited state $\left(\sigma_{1}\right)$ and second excited state absorption cross sections $\left(\sigma_{2}\right)$ for Por-COF-HH, Por-COF-ZnCu and PorCOF-ZnNi (Table S1, Supporting Information). Higher $\beta$ value is observed for metallated porphyrin frameworks (Por-COF-ZnCu and Por-COF-ZnNi) than that of Por-COF-HH (Table S1), which is expected and attributed to the planarity of the metal-porphyrin cores, effective inter-layer stacking, efficient $\pi$-conjugation and successful charge transfer transition between the metal ion's dorbital and the porphyrin $\pi$-orbital. ${ }^{[14,15]}$ The progressive increment of $\beta$ value is in the following order: Por-COF-ZnCu > Por-COF-ZnNi > Por-COF-HH. The detailed intensity dependent open aperture z-scan data for all the three samples are presented in the Supporting Information (Figure S26-28, Section S5). Moreover, the obtained NLO parameters, $\beta$ and the figure of merit $\left(\mathrm{FOM}=\sigma_{1} / \sigma_{0}\right)$ values are as high as $4470 \mathrm{~cm} / \mathrm{GW}$ for Por-COF-ZnCu and $3762 \mathrm{~cm} / \mathrm{GW}$, for Por-COF-ZnNi, respectively, which are found to be higher than those of other reported materials such as metalated porphyrins $(\beta=132-366$ $\mathrm{cm} / \mathrm{GW})$, MOFs $(\beta=0.28-0.46 \mathrm{~cm} / \mathrm{GW})$, graphene $(\beta=900$ $\mathrm{cm} / \mathrm{GW}$ ), etc. (Table S2). ${ }^{[11,16-20]}$ Noteworthy, the observed switching behaviour from SA to RSA is highly interesting to be considered for optical switching applications. ${ }^{[21]}$ Moreover, the switch over from RSA to SA behaviour can be applicable in optical limiting devices based on RSA behaviour at higher intensities and mode locked lasers as it shows SA behaviour at low input intensities.

In summary, three regioregular, crystalline COFs composed of entirely porphyrin units employing Schiff base condensation of A4B4 porphyrin aldehydes and amines have been synthesized. By de novo design, we could systematically embed two different metals $(\mathrm{ZnCu}$ and $\mathrm{ZnNi}$ ) within a crystalline organic framework. Moreover, to prove the concept and to draw the benefits from infinite conjugated porphyrin network, the NLO properties of these Por-COFs were investigated under $532 \mathrm{~nm}$ excitation wavelength using Z-scan technique. Interestingly, rare optical switching behavior from SA to RSA with input intensity was observed for these Por-COFs, while the metalated counterparts (Por-COF- $\mathrm{ZnNi}$ and $-\mathrm{ZnCu}$ ) display high nonlinear absorption coefficient values $(\beta=\sim 4500 \mathrm{~cm} / \mathrm{GW})$, surpassing many state-of-art materials. In brief, we established a structureproperty relationship in A4B4 COF. However, there is much opportunity to be explored at the molecular level that will undoubtedly promote COFs as next generation nonlinear optical switching and optical limiting materials.

Keywords: covalent organic frameworks $\cdot$ microporous

materials $•$ porphyrin $\cdot$ nonlinear optics $\cdot$ regioregular

[1] a) P. N. Prasad, D. J. Williams, Introduction to Nonlinear Optical Effects in Molecules and Polymers, Wiley, New York, USA 1990; b) G. C Baldwin, An Introduction to Nonlinear Optics, Plenum, New York, USA 1996; c) M. O. Senge, M. Fazekas, E. G. A. Notaras, W. J. Blau, M. Zawadzka, O. B. Locos, and E. M. N. Mhuircheartaigh, Adv. Mater 2007, 19, 2737-2774; d) R. Medishetty, J. K. Zare, D. Mayer, M Samoc and R. A. Fischer, Chem. Soc. Rev., 2017, 46, 4976-5004; e) J. Yu, Y. Cui, C. Wu, Y. Yang, Z. Wang, M. O'Keeffe, B. Chen, and G. Qian, Angew. Chem. Int. Ed. 2012, 51, 10542 -10545; f) H. S. Quah, W. Chen, M. K. Schreyer, H. Yang, M. W. Wong, W. Ji, J. J. Vittal, Nat. Commun. 2015, 6: 7954, 1-7.

[2] a) J.-H. Chou, H.S. Nalwa, M.E. Kosal, N.A. Rakow, K.S. Suslick, in: K Kadish, K. Smith, R. Guilard (Eds.), The Porphyrin Handbook, 6, Academic Press, New York, 2000 (Chapter 41); b) K. McEwan, K Lewis, G.Y. Yang, L. L. Chng, Y. W. Lee, W. P. Lau, K. S. Lai, Adv Funct. Mater. 2003, 13, 863; c) M. Calvete, G. Y. Yang, M. Hanack Synth. Metal, 2004, 141, 231; d) G. D. L. Torre, P. Vazquez, F. AgulloLopez, T. Torres, Chem. Rev., 2004, 104, 3723.

[3] a) Y.-L. Liu, Z.-B. Liu, J.-G. Tian, Y. Zhu, J.-Y. Zheng, Optics Commun. 2008, 281, 776-781; b) K. Ogawa, A. Ohashi, Y. Kobuke, K. Kamada, K. Ohta, J. Phys. Chem. B, 2005, 109, 22003; c) R. Bonnett, A Harriman, A.N. Kozyrev, J. Chem. Soc., Faraday Trans., 1992, 88, 763; d) W. J. Su, T. M. Cooper, M. C. Brant, Chem. Mater.,1998, 1212; e) K. J. McEwan, G. Bourhill, J. M. Robertson, H. L. Anderson, J. Nonlinear Opt. Phys. Mater., 2000, 9, 451; f) A. Krivocapic, H. L. Anderson, G. Bourhill, R. Ives, S. Clark, K.J . McEwan, Adv. Mater., 2001, 13, 652.

[4] a) B. A. Tsuda, A. Osuka, Science 2001, 293, 79; b) D. Bonifazi, M. Scholl, F. Song, L. Echegoyen, G. Accorsi, N. Armaroli, F. Diederich, Angew. Chem. Int. Ed. 2003, 42, 4966; c) N. Aratani, A. Osuka, Chem. Rec. 2003, 3, 225; d) I.-W. H. wang, T. Kamada, T. K. Ahn, D. M. Ko, T. Nakamura, A. Tsuda, A. Osuka, D. Kim, J. Am. Chem. Soc. 2004, 126 16 187; e) M. Terazima, H. Shimizu, A. Osuka, J. Appl. Phys. 1997, 81, 2946.

[5] a) X. Feng, L. Liu, Y. Honsho,A. Saeki, S. Seki, S. Irle, Y. Dong, A. Nagai, D. Jiang, Angew. Chem. 2012, 124, 2672; Angew. Chem. Int. Ed. 2012, 51, 2618; b) S. Wan, F. Gandara, A. Asano,H. Furukawa, A. Saeki, S. K. Dey, L. Liao, M. W. Ambrogio, Y. Y. Botros, X. Duan, S. 
Seki, J. F. Stoddart, O. M. Yaghi, Chem. Mater., 2011, 23, 4094; c) S Kandambeth, D. B. Shinde, M. K. Panda, B. Lukose, T. Heine, R. Banerjee, Angew. Chem. Int. Ed. Engl. 2013, 52, 13052-13056; d) H. Sahabudeen, H. Qi, B. A. Glatz, D. Tranca, R. Dong, Y. Hou, T. Zhang, C. Kuttner, T. Lehnert, G. Seifert, U. Kaiser, A. Fery, Z. Zheng, X. Feng, Nature Commun., 2016, 7, 13461, 1-8.

[6] a) A. P. Côté, A. I. Benin, N. W. Ockwig, A. J. Matzger, M. O’Keeffe, O. M. Yaghi, Science 2005, 310, 1166; b) S. Kandambeth, A. Mallick, B. Lukose, M.V. Mane, T. Heine, R. Banerjee, J. Am. Chem. Soc. 2012 134, 19524-19527.

[7] G. Das, B. P. Biswal, S. Kandambeth, V. Venkatesh, G. Kaur, M. Addicoat, T. Heine, S. Verma, R. Banerjee, Chem. Sci. 2015, 6, 39313939.

[8] A.K. Mandal, J. Mahmood, J.-B. Baek, ChemNanoMat., 2017, 3, 373391.

[9] S.Y. Ding, J. Gao, Q. Wang, Y. Zhang, W.G. Song, C.Y. Su, W. Wang, J. Am. Chem. Soc. 2011, 133, 19816-19822.

[10] S. Lin, C. S. Diercks, Y.-B. Zhang, N. Kornienko, E. M. Nichols, Y. Zhao, A. R. Paris, D. Kim, P. Yang, O. M. Yaghi, C. J. Chang, Science, 2015 349, 6253, 1208-1213.

[11] a) P. C. Ray, P. Bonifassi, and J. Leszczynski, J. Phys. Chem. A, 2008 112, 2870-2879; b) D. Swaina, A. Rana, P. K. Panda, S. V. Rao, Chem. Phys. Lett., 2014, 610-611, 310-315.

[12] a) B. Lukose, A. Kuc, T. Heine, Chem. Eur. J. 2011, 17, 2388; b) L. Sarkisov and A. Harrison, Mol. Simulat., 2011, 37, 1248-1257.

[13] M. Gouterman, in: D. Dolphin (Ed.), "The Porphyrins", vol. III, s1, Academic Press, New York, 1978.
[14] a) M. B. M. Krishna, V. P. Kumar, N. Venkatramaiah, R. Venkatesan and D. N. Rao, Appl. Phys. Lett. 2011, 98, 081106; b) M. Sheik-Bahae A. A. Said, T. Wei, D. J. Hagan, E. W. Van Stryland, IEEE J. Quantum Electron. 1990, 26, 760-769; c) V. Sreeramulu, K. K. Haldar, A. Patra, D. N. Rao, Optics and Laser Techn. 2016, 118, 30333; d) M. B. M. Krishna, N. Venkatramaiah, D. Narayana Rao, J. Opt. 2014, 16, 015205.

[15] a) E. B. Fleischer, Acc. Chem. Res. 1970, 3, 3, 105-112; b) L. P. Cook, G. Brewer and W. Wong-Ng, Crystals 2017, 7, 223.

[16] a) M. de Torres, S. Semin, I. Razdolski, J. Xu, J. A. A. W. Elemans, T. Rasing, A. E. Rowan and R. J. M. Nolte, Chem. Commun., 2015, 51 2855; b) J. Qin, T. Wada, H. Sasabe, Mol. Cryst. Liq. Cryst. A 1992, 217, 47; c) S. Priyadarshy, M. J. Therien, D. N. Beratan, J. Am. Chem. Soc. 1996, 118, 1504; d) I. D. L. Albert, T. J. Marks, M. A. Ratner, Chem. Mater. 1998, 10, 753.

[17] J. Li, D. Jia, S. Meng, J. Zhang, M. P. Cifuentes, M. G. Humphrey, and C. Zhang, Chem. Eur.J .2015, 21, 7914-7926

[18] X. Jiang, L. Zhang, S. Liu, Y. Zhang, Z. He, W. Li, F. Zhang, Y. Shi, W. Lü, Y. Li, Q. Wen, J. Li, J. Feng, S. Ruan, Y.-J. Zeng, X. Zhu, Y. Lu, and H. Zhang, Adv. Opt. Mater. 2018, 1800561.

[19] X. Li, Q. Gao, J. Aneesh, H.-S. Xu, Z. Chen, W. Tang, C. Liu, X. Shi, K V. Adarsh, Y. Lu, and K. Pi. Loh, Chem. Mater. 2018, 30, 5743-5749.

[20] M. B. M. Krishna, N. Venkatramaiah, R. Venkatesan, and D. N. Rao, J. Mat. Chem., 2012, 22, 3059.

[21] F. Castet, V. Rodriguez†, J.-L. Pozzo, L. Ducasse, A. Plaquet, and B. Champagne, Acc. Chem. Res., 2013, 46, 2656-2665. 


\section{COMMUNICATION}

In this work, we demonstrated three regioregular porphyrin based porous COFs, Por-COF-HH and its dual metalated congeners (Por-COF-ZnCu and Por-COF-ZnNi) with excellent NLO properties. Remarkably, the NLO switching and high parameter values were obtained for these Por-COFs, which is highly demanding for optical switching and optical limiting devices.

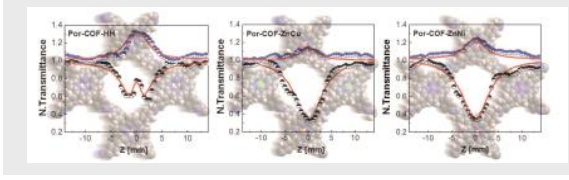

Bishnu P. Biswal, * Sreeramulu Valligatla, Mingchao Wang, Tanmay Banerjee, Nabil Momen, M. Bala Murali Krishna Mariserla, Naisa

Chandrasekhar, Daniel Becker, Matthew Addicoat, Irena Senkovska, Reinhard Berger, D. Narayana Rao, Xinliang Feng*

Page No. - Page No.

Nonlinear Optical Switching in Regioregular Porphyrin Covalent Organic Frameworks 